\title{
Rectangular waveguide-to-coplanar waveguide transitions at U-band using e-plane probe and wire bonding
}

Dong, Yunfeng; Johansen, Tom Keinicke; Zhurbenko, Vitaliy; Hanberg, Peter Jesper

Published in:

Proceedings of the 46th European Microwave Conference

Link to article, DOI:

10.1109/EuMC.2016.7824263

Publication date:

2016

Document Version

Peer reviewed version

Link back to DTU Orbit

Citation (APA):

Dong, Y., Johansen, T. K., Zhurbenko, V., \& Hanberg, P. J. (2016). Rectangular waveguide-to-coplanar waveguide transitions at U-band using e-plane probe and wire bonding. In Proceedings of the 46th European Microwave Conference (pp. 5-8). IEEE. https://doi.org/10.1109/EuMC.2016.7824263

\section{General rights}

Copyright and moral rights for the publications made accessible in the public portal are retained by the authors and/or other copyright owners and it is a condition of accessing publications that users recognise and abide by the legal requirements associated with these rights.

- Users may download and print one copy of any publication from the public portal for the purpose of private study or research.

- You may not further distribute the material or use it for any profit-making activity or commercial gain

- You may freely distribute the URL identifying the publication in the public portal 


\section{Rectangular Waveguide-to-Coplanar Waveguide Transitions at U-Band Using E-Plane Probe and Wire Bonding}

\author{
Yunfeng Dong, Tom K. Johansen, Vitaliy Zhurbenko \\ Electromagnetic System, Department of Electrical Engineering \\ Technical University of Denmark \\ DK-2800 Kgs. Lyngby, Denmark \\ yundon/tkj/vz@elektro.dtu.dk
}

\author{
Peter Jesper Hanberg \\ DTU Danchip \\ Technical University of Denmark \\ DK-2800 Kgs. Lyngby, Denmark \\ jehan@danchip.dtu.dk
}

\begin{abstract}
This paper presents rectangular waveguide-tocoplanar waveguide (CPW) transitions at U-band $(40$ - $60 \mathrm{GHz})$ using E-plane probe and wire bonding. The designs of CPWs based on quartz substrate with and without aluminum cover are explained. The single and double layer rectangular waveguideto-CPW transitions using E-plane probe and wire bonding are designed. The proposed rectangular waveguide-to-CPW transition using wire bonding can provide $10 \mathrm{GHz}$ bandwidth at $\mathrm{U}$ band and does not require extra CPWs or connections between CPWs and chips. A single layer rectangular waveguide-to-CPW transition using E-plane probe with aluminum package has been fabricated and measured to validate the proposed transitions. To the authors' best knowledge, this is the first time that a wire bonding is used as a probe for rectangular waveguide-to-CPW transition at U-band.
\end{abstract}

\section{INTRODUCTION}

Since rectangular waveguide was first invented, it has continuously been used as a transmission line for a wide range of applications. This is due to low loss, high powerhandling capability, and its simple structure. The components and chips used in microwave integrated circuits (MICs) as well as monolithic microwave integrated circuits (MMICs) mainly rely on planar structures due to fabrication processes. As a result, the transitions between waveguides and planar transmission lines are needed, in particular rectangular waveguide-toCPW transitions, which are widely used for system integration and packaging at millimeter-wave and submillimeter-wave frequencies [1], [2]. Different types of transitions have been designed to guide electromagnetic waves between rectangular waveguides and planar transmission lines. According to [3], the transitions could be a ridge waveguide in-line transition, a trough waveguide transition, a rectangular waveguide transition with a tapered ridge, a rectangular waveguide end launcher, or a rectangular waveguide transition with a printed probe. Fig. 1 shows the basic structure of a rectangular waveguide-to-CPW transition using an E-plane probe. In this type of transition the signal trace of the $\mathrm{CPW}$ is extended into the rectangular waveguide forming a rectangular patch which is expected to provide a probe for wideband transmissions.

Rectangular waveguide-to-CPW transitions using E-plane probe and wire bonding are described in this paper. In Section II, the designs of CPWs based on quartz substrate with and without aluminum cover for transitions at U-band $(40-60$

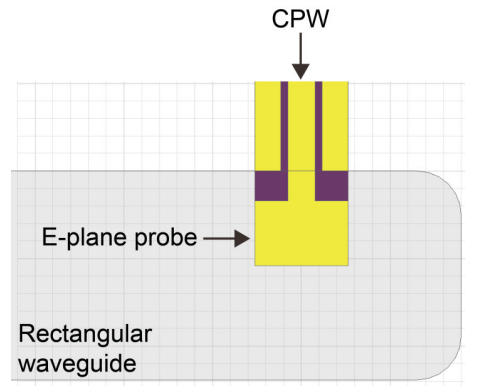

Fig. 1. Rectangular waveguide-to-CPW transition using E-plane probe

$\mathrm{GHz}$ ) are explained. In Section III, the proposed single and double layer rectangular waveguide-to-CPW transitions using E-plane probe and wire bonding are described. In Section IV, the fabrication and measurement results of a single layer rectangular waveguide-to-CPW transition using E-plane probe based on quartz substrate and aluminum package are shown.

\section{Design Procedure of Coplanar Waveguide}

CPW has been widely used since it was demonstrated in 1969 by C.P. Wen [4]. In a CPW, two ground traces are located on both sides of the signal trace forming a groundsignal-ground (GSG) structure and a quasi-TEM mode can be supported. Compared to conventional microstrip line, $\mathrm{CPW}$ is more versatile. For specified substrate properties and circumstances, the characteristic impedance of the CPW is mainly determined by the ratio of $W /(W+2 G)$. $W$ is the width of the signal trace while $G$ is the width of the gap between the signal and ground traces. With this special characteristic, the size of CPW can be tuned for applications and requirements instead of being restricted by the substrates. This also makes CPW suitable for transitions between structures on different substrates since it can be tapered in or out to fit the connections without affecting the characteristic impedance. In [5], the performance of CPW has been proved for high frequency integration and packaging up to $300 \mathrm{GHz}$.

Fig. 2 shows the simulation structure of a CPW based on quartz substrate which is designed to test its performance from $40 \mathrm{GHz}$ to $60 \mathrm{GHz}$ (U-band). The width of the signal trace is $100 \mu \mathrm{m}$ and the width of the ground trace is $300 \mu \mathrm{m}$ with a gap width of $30 \mu \mathrm{m}$. The material of the trace conductors is 


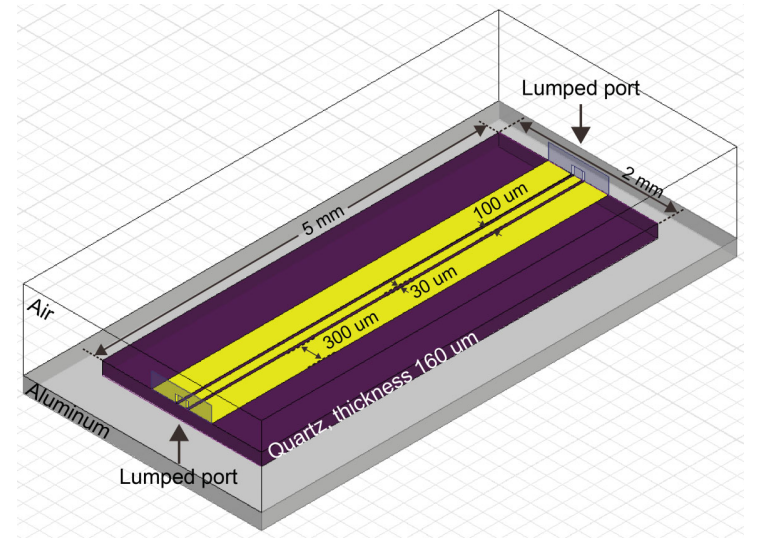

Fig. 2. Simulation structure of CPW using lumped ports in HFSS.

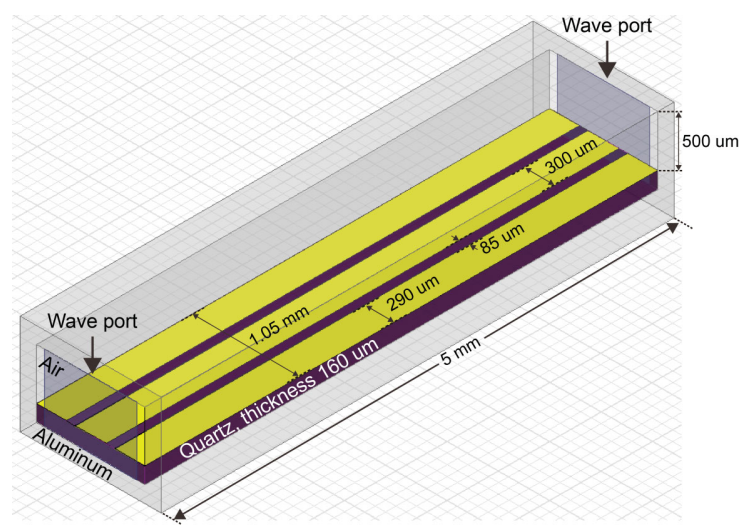

Fig. 3. Simulation structure of CPW inside aluminum cover using wave ports in HFSS.

gold. Taking into account the skin depth effect, the thickness of the conductor is $400 \mathrm{~nm}$ which is more than the skin depth at the lower limit frequency range of the interest. The material of the substrate is quartz which, according to [6], is one of the commonly used materials for waveguide transitions. The substrate is $5 \mathrm{~mm}$ in length and $2 \mathrm{~mm}$ in width with a thickness of $160 \mu \mathrm{m}$.

High Frequency Structural Simulator (HFSS) is used in this work for 3D full-wave electromagnetic simulations. As it shown in Fig. 2, an air box and an aluminum base are included in the simulation for accuracy improvement. Lumped ports with vertical perfect electric conductor (PEC) bridges are used as the excitation scheme. Different excitation schemes in HFSS for CPW structures are described and compared in [7]. The red solid lines in Fig. 4 show the simulation results of the designed $\mathrm{CPW}$ up to $80 \mathrm{GHz}$. In the frequency range of U-band, the return loss remains better than $15 \mathrm{~dB}$ while the insertion loss is less than $0.8 \mathrm{~dB}$.

As it shown in Fig. 1, the CPW of the transition structure is usually packaged inside an aluminum box, which changes the behaviours of the conventional CPW. Fig. 3 shows the simulation structure of a CPW packaged inside an aluminum box. The width of the signal trace is $300 \mu \mathrm{m}$ and the width of the ground trace is $290 \mu \mathrm{m}$ with a gap width of $85 \mu \mathrm{m}$. The material of the trace conductors is gold with a thickness of 400 $\mathrm{nm}$. The length of the quartz substrate is $5 \mathrm{~mm}$ while the width is reduced to $1.05 \mathrm{~mm}$ in order to suppress the parasitic modes

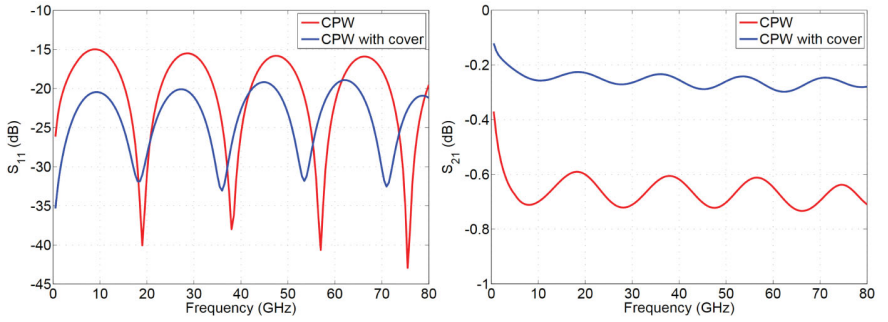

Fig. 4. Simulation results of CPW with (blue solid lines) and without (red solid lines) aluminum cover.

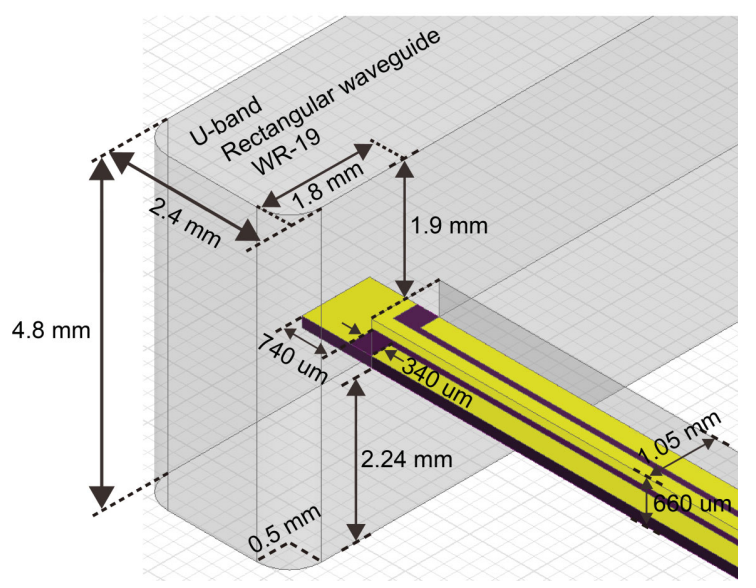

Fig. 5. Single layer rectangular waveguide-to-CPW transition using E-plane probe.

caused by the aluminum cover. The height of the air cavity is $500 \mu \mathrm{m}$. Wave ports are used as the excitation scheme in the simulation. The blue solid lines in Fig. 4 show the simulation results of the designed CPW with aluminum cover. The return loss is better than $20 \mathrm{~dB}$ and the insertion loss is less than 0.3 $\mathrm{dB}$ in the frequency range of U-band.

\section{DESIGN PROCEDURE OF RECTANGULAR WAVEGUIDE-TO-CPW TRANSITIONS}

\section{A. Transitions Using E-Plane Probe}

Fig. 5 shows a single layer rectangular waveguide-to$\mathrm{CPW}$ transition using E-plane probe. In this design, U-band rectangular waveguides (WR-19) are used and the radius of the round corners at the end of the waveguides is 500 um due to machining process. A window is opened in parallel with the $\mathrm{H}$-plane of the waveguide and connected to an air cavity. The substrate is placed inside the air cavity and plugged into the waveguide. The CPW and substrate are the same as shown in Fig. 3. At the end of the CPW, the signal trace connects to a rectangular patch which works as a probe. The probe is in parallel with the E-plane of the waveguide and it is called an E-plane probe transition.

The E-plane probe is located $1.8 \mathrm{~mm}$ from the end of the waveguide which distance is around $\lambda / 4$ at $45 \mathrm{GHz}$. This works as a $\lambda / 4$ impedance transformer where a short termination transforms into an open termination. The width and length of the E-plane probe are $1.05 \mathrm{~mm}$ and $740 \mu \mathrm{m}$ respectively. The gap between the probe and the CPW is 340 $\mu \mathrm{m}$. The size of the probe is mainly determined by frequency 


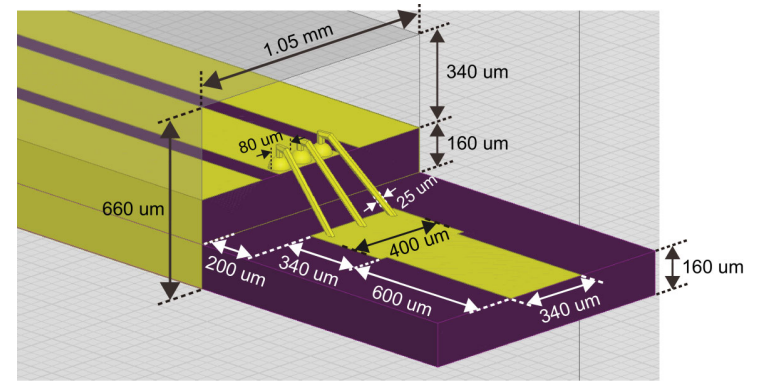

Fig. 6. Double layer rectangular waveguide-to-CPW transition using E-plane probe.
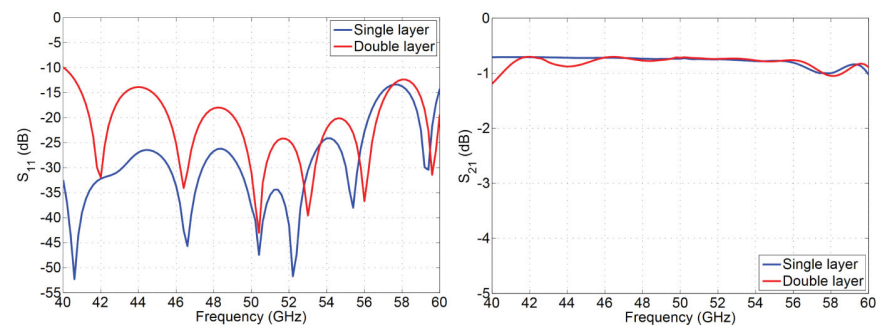

Fig. 7. Simulation results of single (blue solid lines) and double (red solid lines) layer transitions using E-plane probe.

and substrate. By changing the size, the probe can be easily configured for other frequency bands which makes it versatile.

The E-plane probe transition shown in Fig. 5 is simulated in HFSS. The simulation structure is symmetric and contains two transitions. The length of the CPW is $2 \mathrm{~cm}$ and the length of the input/output WR-19 waveguide is $5 \mathrm{~cm}$. The blue solid lines in Fig. 7 show the simulation results of the designed rectangular waveguide-to-CPW transition at U-band. The return loss remains better than $10 \mathrm{~dB}$ while the insertion loss is less than $1 \mathrm{~dB}$.

When E-plane probe transitions are used for high frequency integration and packaging, it becomes critical to realize good connections between the CPWs and chips. Under this circumstance, it would be better if the E-plane probe can be directly connected to the chip or even built on the chip [8]. Fig. 6 shows a double layer rectangular waveguide-to-CPW transition using E-plane probe. The bottom layer contains the pattern of the probe and also works as a base for the chip providing ground connections and physical support. The top layer is a CPW which can be replaced by other chips for different applications. The E-plane probe is connected to the CPW through wire bondings. In this way, the probe is directly connected to the chip. The probe pattern is optimized to compensate for the parasitic inductance of the wire bondings. The red solid lines in Fig. 7 show the simulation results of the double layer rectangular waveguide-to-CPW transition at U-band. Although the performance of the double layer transition degrades a little bit in comparison with the single layer E-plane probe transition, it still provides a wideband transmission.

\section{B. Transitions Using Wire Bonding}

Since wire bondings are used for connections between CPWs and chips or between E-plane probes and chips, it would be better if the wire bonding itself works as a probe. Fig. 8 shows a single layer rectangular waveguide-to-CPW

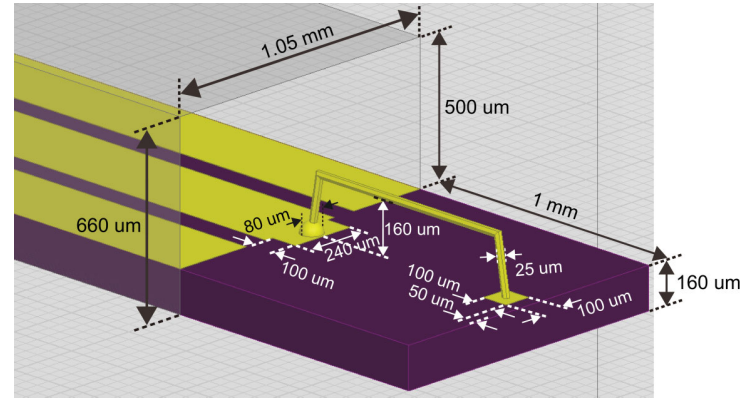

Fig. 8. Single layer rectangular waveguide-to-CPW transition using wire bonding.

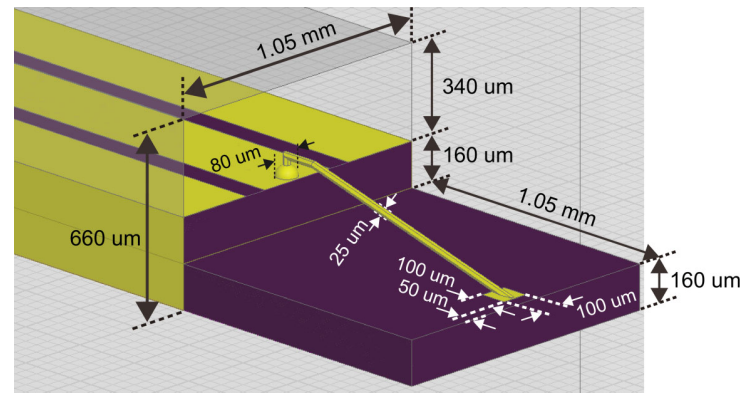

Fig. 9. Double layer rectangular waveguide-to-CPW transition using wire bonding.

transition using wire bonding. The substrate is inserted into the rectangular waveguide and there is a small square pad at the end of the substrate which is used for connecting wire bonding from the CPW. The length of the square pad is $100 \mu \mathrm{m}$ and locates $50 \mu \mathrm{m}$ from the edge of the substrate. The signal trace of CPW is extended $100 \mu \mathrm{m}$ with a width of $240 \mu \mathrm{m}$ for connecting wire bondings. The blue solid lines in Fig. 10 show the simulation results of the rectangular waveguide-toCPW transition using wire bonding at U-band. In comparison with the designed E-plane probe transition, it has a narrower bandwidth of $7 \mathrm{GHz}$ at U-band. From $45 \mathrm{GHz}$ to $52 \mathrm{GHz}$, the return loss remains better than $10 \mathrm{~dB}$ while the insertion loss is less than $2 \mathrm{~dB}$. In Fig. 8, the height of the wire bonding is $160 \mu \mathrm{m}$ and the length is $850 \mu \mathrm{m}$. A bandwidth of $7 \mathrm{GHz}$ is achieved and the passband can be tuned by changing the height and length of the wire bonding. Unlike E-plane probe transitions, wire bonding transitions have simpler structure and it can be used for narrowband applications in which the input and output are only at specific frequencies.

A double layer rectangular waveguide-to-CPW transition using wire bonding is shown in Fig. 9. In order to prove the possibility of using wire bonding transitions for chips, a CPW is placed on the top layer which represents the chip and it is directly connected to the square pad on the bottom layer through a wire bonding. The length and height of the wire bonding are $1 \mathrm{~mm}$ and $190 \mu \mathrm{m}$ respectively which are optimized so that the passband locates around the middle of U-band.

The red solid lines in Fig. 10 show the simulation results of the double layer rectangular waveguide-to-CPW transition using wire bonding. A bandwidth of $10 \mathrm{GHz}$ is achieved and the passband ranges from $46 \mathrm{GHz}$ to $56 \mathrm{GHz}$. The return loss is better than $10 \mathrm{~dB}$ and the insertion loss is less than $2 \mathrm{~dB}$. In 

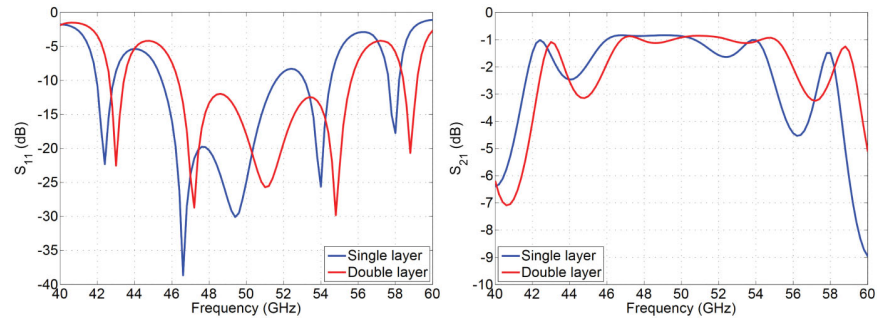

Fig. 10. Simulation results of single (blue solid lines) and double (red solid lines) layer transitions using wire bonding.

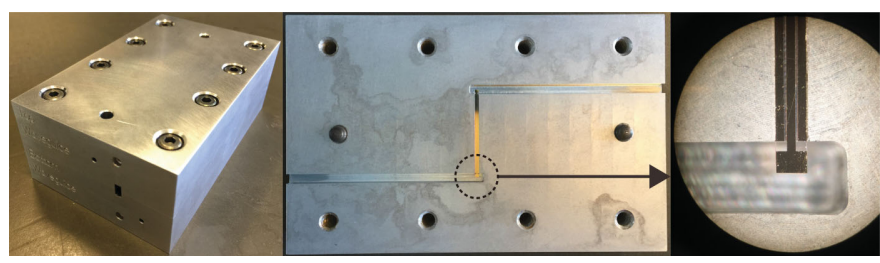

Fig. 11. Fabricated rectangular waveguide-to-CPW transition using E-plane probe.

comparison with the conventional E-plane probe transition in Fig. 5, the integration is simpler using wire bonding transition which does not contain extra CPWs or wire bondings between CPWs and chips.

\section{FABRICATION AND EXPERIMENTAL RESUlTS}

In order to prove the designs, the single layer rectangular waveguide-to-CPW transition using E-plane probe shown in Fig. 5 was fabricated. The E-plane probe transition based on quartz substrate was made in Danchip (National Center for Micro- and Nanofabrication in Denmark). The thickness of the gold conductors is $400 \mathrm{~nm}$ and there is a titanium layer below with a thickness of $30 \mathrm{~nm}$ improving the adhesion of the gold. Both layers were deposited using sputtering. The patterning of the metal layers was done by laser ablation using a picosecond laser with a wavelength of $355 \mathrm{~nm}$ focused down to a spot size of approximate $10 \mu \mathrm{m}$. The aluminum package was made inhouse and it was cut into two parts. The bottom part contains the input and output WR-19 waveguides with a $160 \mu \mathrm{m}$ deep slot in the middle. The E-plane probe transition was put in the slot. Silver conductive glue was used to fill the gap between the substrate and the slot which provides ground connections to the CPW. The top part contains the input and output WR-19 waveguides with a $500 \mu \mathrm{m}$ deep slot in the middle. The slot works as an air cavity. Fig. 11 shows the fabricated transition and its aluminum package.

A network analyzer (Agilent E8361A) and two waveguideto-coax adapters (FM 24094-VF50) were used to test the fabricated transition. A waveguide calibration kit (Agilent U11644A) was used to calibrate out the effects of the cables and adapters. The measurement results are shown in Fig. 12 and compared with the simulation results using HFSS. The return loss is better than $10 \mathrm{~dB}$ from $40 \mathrm{GHz}$ to $58 \mathrm{GHz}$ and it goes to $8.5 \mathrm{~dB}$ from $58 \mathrm{GHz}$ to $60 \mathrm{GHz}$. The insertion loss is less than $3 \mathrm{~dB}$ in the frequency band. The differences between the measurement and simulation are caused by the tolerance of manufacturing, the ground connections between the CPW and the aluminum package, and the junctions between the top and bottom part of the waveguide.
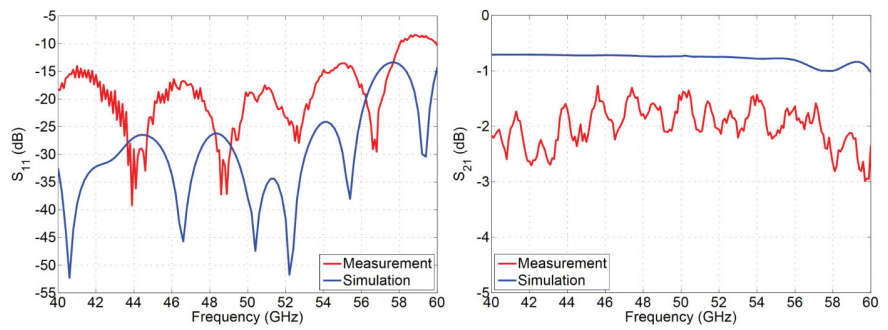

Fig. 12. Measurement (red solid lines) and simulation (blue solid lines) results of the rectangular waveguide-to-CPW transition using E-plane probe.

\section{CONClusion}

Rectangular waveguide-to-CPW transitions at U-band using E-plane probe and wire bonding have been presented. The fundamental properties and packaging effects of the CPWs based on quartz substrate at U-band were described. Single and double layer transitions using E-plane probe and wire bonding have been designed. It was shown that E-plane probe transitions can support higher bandwidth while the integration is simpler for wire bonding transitions which does not contain extra CPWs or connections between CPWs and chips. The designed transitions were optimized for U-band and can be easily reconfigured to other frequency bands. A bandwidth of $10 \mathrm{GHz}$ was shown for the proposed double layer wire bonding transitions at U-band which can be tuned for different applications. To the authors' best knowledge, this is the first time that a wire bonding probe is used for rectangular waveguide-to-CPW transition at U-band. The designed single layer transition using E-plane probe with an aluminum package has been fabricated and measured to validate the proposed transitions.

\section{REFERENCES}

[1] T. Reck, R.M. Weikle, and N.S. Barker. A waveguide to unenclosed coplanar waveguide transition. Microwave Theory and Techniques, IEEE Transactions on, 58(9):2420-2425, Sept 2010.

[2] K.M.K.H. Leong, W.R. Deal, V. Radisic, Xiao Bing Mei, J. Uyeda, L. Samoska, A. Fung, T. Gaier, and R. Lai. A 340-380 GHz integrated CBCPW-to-waveguide transition for sub millimeter-wave MMIC packaging. Microwave and Wireless Components Letters, IEEE, 19(6):413-415, June 2009.

[3] N. S. Rainee. Coplanar waveguide circuits, components and systems. Wiley, 2001.

[4] C.P. Wen. Coplanar waveguide: A surface strip transmission line suitable for nonreciprocal gyromagnetic device applications. Microwave Theory and Techniques, IEEE Transactions on, 17(12):1087-1090, Dec 1969.

[5] K. Eriksson, P.J. Sobis, S.E. Gunnarsson, J. Hanning, and H. Zirath. InP DHBT amplifier modules operating between 150-300 GHz using membrane technology. Microwave Theory and Techniques, IEEE Transactions on, 63(2):433-440, Feb 2015.

[6] L.A. Samoska. An overview of solid-state integrated circuit amplifiers in the submillimeter-wave and $\mathrm{THz}$ regime. Terahertz Science and Technology, IEEE Transactions on, 1(1):9-24, Sept 2011.

[7] Yunfeng Dong, Tom K. Johansen, Vitaliy Zhurbenko, Antonio Beretta, Antonello Vannucci, and Gianpietro Locatelli. A 3D hybrid integration methodology for terabit transceivers. In Microwave and Optoelectronics Conference (IMOC), 2015 SBMO/IEEE MTT-S International, pages 1-5, Nov 2015.

[8] T. Merkle, R. Gotzen, Joo-Young Choi, and S. Koch. Polymer multichip module process using 3-D printing technologies for D-band applications. Microwave Theory and Techniques, IEEE Transactions on, 63(2):481-493, Feb 2015. 